\title{
Classical and Nonclassical Levy Laplacians
}

\author{
L. Accardi ${ }^{a}$ and O. G. Smolyanov ${ }^{b}$ \\ Presented by Academician V.S. Vladimirov March 1, 2007
}

Received March 5, 2007

DOI: $10.1134 / \mathrm{S} 1064562407060014$

Comparatively recently, it was discovered that the Yang-Mills equations are equivalent to the Laplace equation with Laplacian virtually coinciding with the operator introduced at the beginning of the past century by Paul Levy (see [1-3] and the references therein). In [1-3], some modifications of the Laplace-Levy operators were also introduced. In what follows, we refer to the operators introduced by Levy as classical Levy Laplacians and to their modifications, as nonclassical Laplacians. In this paper, we describe a general method for defining and studying both types of operators, which makes it possible to extend results on Levy Laplacians to nonclassical Laplacians (of course, with natural modifications).

We define an infinite family of Laplacians, whose elements are classical Levy Laplacians and the nonclassical Laplacian related to the Yang-Mills equations (it is natural to call the remaining elements nonclassical Laplacians of corresponding orders) and describe the relationship between these Laplacians and quantum random processes. The suggested constructions use functionals on a space of operators, which we call Cesàro traces and define by using suitable Cesàro means. Simultaneously, we consider Volterra Laplacians and find analogies between them and Levy Laplacians.

\section{DEFINITIONS AND TERMINOLOGY}

Let $E$ be a separable locally convex space (LCS) admitting continuous Hilbert norms; suppose that $p$ is one of such norms, $H$ is the Hilbert space obtained by completing $E$ with respect to the norm $p$, and $(\cdot, \cdot)_{H}$ is inner product in this space; we denote the norm generated by inner product by the same symbol $p$. Suppose also that $E^{*}$ is the space of continuous linear functionals on $E$ endowed with the locally convex topology compatible with the duality between $E^{*}$ and $E$. Then,

\footnotetext{
a University of Rome “Tor Vergata," Rome, Italy

${ }^{b}$ Moscow State University, Leninskie gory, Moscow, 119991 Russia
}

the canonical embedding of the LCS $E$ into the Hilbert space $H$ is continuous and has dense image (in fact, this image is $E$ itself). It follows that the mapping $H^{*} \rightarrow E^{*}$ dual to this embedding ( $H^{*}$ denotes the Hilbert dual to the space $H$ ) is also a continuous embedding with dense image. Therefore, identifying the spaces $H$ and $H^{*}$, we obtain a rigged Hilbert space $E \subset H=H^{*} \subset E^{*} .{ }^{1}$ If $K$ is an LCS, then the expression $E \subset K \subset E^{*}$ means that the corresponding embeddings are continuous. Note that if $x \in E$ and $g \in H \subset E^{*}$, then we have, in natural notation, $(x, g)_{H}=\langle g, x\rangle(\equiv g(x))$. If $g \in E^{*}$ and $x \in E^{*}$, then the symbol $\langle g, x\rangle$ is also used in the case where $x$ belongs to the completion of $E$ in the continuous norm with respect to which the functional $g$ is continuous; in this case, $\langle g, x\rangle$ denotes the value at $x$ of the extension of $g$ to this completion.

Finally, let $\mathbf{e}=\left(e_{n}\right)$ be an orthonormal basis in $H$ consisting of elements of the space $E$. We assume that the linear span of the basis $\mathbf{e}$ is dense in $E$. The objects defined in what follows depend on the choice of the norm $p$ (which determines the embedding of $E$ into $E^{*}$ ) and of the basis $\mathbf{e}$, but we do not mention this as a rule.

The further exposition is partly formal, and some analytic assumptions are omitted.

\section{TRACES AND CESÀRO INNER PRODUCTS}

For LCS $G_{1}$ and $G_{2}$, the symbol $L\left(G_{1}, G_{2}\right)$ denotes the vector space of all linear continuous mappings from $G_{1}$ to $G_{2}$; instead of $L(G, G)$, we write $L(G)$. The terms "operator" and "linear mapping" are considered as synonyms; thus, an operator is a linear mapping from one LCS to another. Let $D$ be a linear mapping from $E$ to $E^{*}$.

\footnotetext{
${ }^{1}$ In the definition of a rigged Hilbert space, it is usually assumed in addition that $E$ is a nuclear countably Hilbert space, i.e., $E$ is a nuclear Fréchet space whose topology is determined by a (countable) family of pairwise comparable compatible Hilbert norms. Note that there exist non-countably Hilbert nuclear Fréchet spaces admitting continuous (Hilbert) norms.
} 
Definition 1. If $A \in L\left(E, E^{*}\right)$, then the trace $\operatorname{tr}_{D} A$ of an operator $A$ generated by an operator $D$ is defined as

$$
\operatorname{tr}_{D} A=\sum_{j=1}^{\infty}\left\langle A D e_{j}, D e_{j}\right\rangle .
$$

In particular, if $A$ is the restriction to $E$ of an operator $A_{0} \in L(H)$ and $D$ is the identity mapping (on the space $E$ ), then $\operatorname{tr}_{D} A$ coincides with the usual trace of the operator $A_{0}$ on the Hilbert space $H$.

Definition 2. If $A \in L\left(E, E^{*}\right)$, then the Cesàro trace $\operatorname{tr}_{D}^{C} A$ of an operator $A$ generated by an operator $D$ is defined as

$$
\operatorname{tr}_{D}^{C} A=\lim _{k \rightarrow \infty} \frac{1}{k} \sum_{j=1}^{k}\left\langle A D e_{j}, D e_{j}\right\rangle .
$$

In particular, the Cesàro trace generated by the identity operator coincides with the Levy trace defined in [4].

Remark 1. If $A$ is a linear operator on a Hilbert space $H$ and $\left(e_{n}\right)$ is an orthonormal basis in $H$ contained in the domain of $A$, then the Cesàro trace $\operatorname{tr} A$ of the operator $A$ on $H$ (with respect to the basis $\left(e_{n}\right)$ ) is defined by

$$
\operatorname{tr} A=\lim _{k \rightarrow \infty} \frac{1}{k} \sum_{j=1}^{k}\left(A e_{n}, e_{n}\right),
$$

where (.) is inner product in $H$. Thus, if $E=H=H^{*}=$ $E^{*}, \mathbf{e}=\left(e_{n}\right)$, and $A \in L(H)$, then this Cesàro trace coincides with the above Cesàro trace generated by the identity operator.

Remark 2. Cesàro traces generated by nonidentity operators are said to be nonclassical. In fact, we used such traces in [4] to define operators which we called exotic Levy Laplacians.

Definition 3. The inner product generated by an operator $D$ is the function denoted by $(\cdot, \cdot)_{D}$ and defined as follows. The domain $\operatorname{dom}(\cdot, \cdot)_{D}$ of this function is the Cartesian square of the vector subspace

$$
\left\{a \in E^{*}: \sum_{j=1}^{\infty}\left|\left\langle a, D e_{j}\right\rangle\right|^{2}<\infty\right\}
$$

of the space $E^{*}$; if $(a, b) \in \operatorname{dom}(\cdot, \cdot)_{D}$, then

$$
(a, b)_{D}=\sum_{j=1}^{\infty}\left\langle a, D e_{j}\right\rangle\left\langle b, D e_{j}\right\rangle
$$

Definition 4. The Cesàro inner product generated by an operator $D$ is the function denoted by $(\cdot, \cdot)_{D}^{C}$ and defined as follows. The domain of this function is the subset $\operatorname{dom}(\cdot, \cdot)_{D}^{C}$ of the space $E^{*} \times E^{*}$ determined by the equality $\operatorname{dom}(\cdot, \cdot)_{D}^{C}=\left\{(a, b) \in E^{*} \times E^{*}\right.$ : the sequence $\left(\left\langle a, D e_{j}\right\rangle\left\langle b, D e_{j}\right\rangle\right)$ has Cesàro mean $\}$; if $(a, b) \in$ $\operatorname{dom}(\cdot, \cdot)_{D}^{C}$, then $(a, b)_{D}^{C}=\lim _{k \rightarrow \infty} \frac{1}{k} \sum_{j=1}^{k}\left\langle a, D e_{j}\right\rangle\left\langle b, D e_{j}\right\rangle$.

Remark 3. It can be shown that the set of those $a \in$ $E^{*}$ for which the Cesàro inner square $(a, a)_{D}^{C}$ is defined (and finite) is not a linear space; therefore, for Cesàro inner product, the analogue of Definition 3 is incorrect.

Example 1. If $a, b \in E^{*}$ and $A=a \otimes b$, then $\operatorname{tr}_{D}^{C} A=$ $(a, b)_{D}^{C}$.

\section{LAPLACE-LEVY AND LAPLACE-VOLTERRA OPERATORS}

In this section, we define the operators mentioned in the title and consider their simplest properties. The exposition is organized so as to emphasize the similarity between these operators rather than the difference between them. In the same way as usual traces (including those generated by nonidentity operators) determine Volterra Laplacians (which are known also as Gross Laplacians), Cesàro traces determine LaplaceLevy operators.

A real-valued function $f$ on $E$ is said to be differentiable in a direction $h \in E^{*} \backslash E$ at a point $x \in E$ if it is Gáteaux differentiable at this point and there exists an LCS $K$ such that $E \subset K \subset E^{*}, h \in K$, and the derivative $f^{\prime}(x)$ is continuous in the topology induced on $E$ by the topology of the space $K$. In this case, the derivative of the function $f$ in the direction $h \in E^{*} \backslash E$ at the point $x \in$ $E$ is defined as the value at $h$ of the continuous extension of $f^{\prime}(x)$ to $K$; this derivative is denoted by $f^{\prime}(x) h$. Let $C_{2}(E)$ be the vector space of all twice Gáteaux differentiable (real-valued) functions on $E$; by $\operatorname{dom}_{D}$ we denote the vector subspace of this space consisting of all functions $f$ such that, for every $x \in E, \operatorname{tr}_{D} f^{\prime \prime}(x)$ exists, and $\operatorname{dom}_{D}^{L}$ is the vector subspace consisting of all functions $f$ such that, for every $x \in E, \operatorname{tr}_{D}^{C} f^{\prime \prime}(x)$ exists.

Definition 5. The Laplace-Levy operator on $C_{2}(E)$ determined by an operator $D$ is the (linear) mapping $\Delta_{D}^{L}$ from $\operatorname{dom}_{D}^{L}$ to the function space on $E$ defined by

$$
\left(\Delta_{D}^{L} f\right)(x)=\operatorname{tr}_{D}^{C} f^{\prime \prime}(x) .
$$


The Levy-Laplace operator determined by the identity operator is said to be classical; it is denoted by $\Delta^{L}$.

Definition 6. The Laplace-Volterra operator on $C_{2}(E)$ determined by an operator $D$ is the (linear) mapping $\Delta_{D}$ from $\operatorname{dom}_{D}$ to the function space on $E$ defined by

$$
\left(\Delta_{D} f\right)(x)=\operatorname{tr}_{D} f^{\prime \prime}(x) .
$$

Remark 4. The above definitions of operators depend on the choice of the norm $p$ determining the rigged Hilbert space structure, although the notation does not indicate this.

Remark 5. The Laplace-Volterra operators determined by nonidentity operators $D$ have been considered earlier, although, apparently, their properties discussed below have not been mentioned explicitly.

Many properties of Laplace-Levy and Laplace-Volterra operators determined by nonidentity operators are similar to those of operators determined by the identity operator, which were described in [4]. In this paper, we state only two propositions.

Proposition 1 (the chain rule). If $g \in C^{2}(E)$ and $f \in$ $C^{2}\left(\mathbb{R}^{1}\right)$, then

$$
\begin{gathered}
\Delta_{D}^{L}(f \circ g)(x)=f^{\prime \prime}(g(x))\left(g^{\prime}(x), g^{\prime}(x)\right)_{D}^{C} \\
+f^{\prime}(g(x))\left(\Delta_{D}^{L} g\right)(x)
\end{gathered}
$$

and

$$
\begin{gathered}
\Delta_{D}(f \circ g)(x)=f^{\prime \prime}(g(x))\left(g^{\prime}(x), g^{\prime}(x)\right)_{D} \\
+f^{\prime}(g(x))\left(\Delta_{D} g\right)(x) .
\end{gathered}
$$
then

Proposition 2 (Leibniz' formula). If $g, f \in C^{2}(E)$,

$$
\begin{aligned}
\Delta_{D}^{L}(f \cdot g)(x) & =g(x) \cdot \Delta_{D}^{L} f(x)+f(x) \cdot \Delta_{D}^{L} g(x) \\
& +2\left(f^{\prime}(x), g^{\prime}(x)\right)_{D}^{C}
\end{aligned}
$$

and

$$
\begin{aligned}
\Delta_{D}(f \cdot g)(x) & =g(x) \cdot \Delta_{D} f(x)+f(x) \cdot \Delta_{D} g(x) \\
& +2\left(f^{\prime}(x), g^{\prime}(x)\right)_{D} .
\end{aligned}
$$

The following proposition and example describe a relationship between the classical and nonclassical Laplace-Levy operators. Their statements do not include any assumptions about the domains of these operators.

Proposition 3. If $F \in C_{2}(E)$, then $\Delta^{L} F(x)=\Delta_{D}^{L}(F \circ$ $\left.D^{-1}\right)(x)$.

Example 2. Suppose that $H=L_{2}(0, \pi), E$ is the space of infinitely differentiable functions on $[0, \pi]$ vanishing at zero endowed with the topology of uniform convergence of functions and their derivatives of any order, and the elements of an orthonormal basis e are determined by the equalities $e_{n}(t)=\sqrt{\frac{2}{\pi}} \sin t$. Suppose also that functions $F_{j}(j=1,2)$ on $E$ and an operator $D$ are defined by

$$
\begin{gathered}
F_{1}(x)=\int_{0}^{\pi} f_{1}(t)(x(t))^{2} d t, \\
F_{2}(x)=\int_{0}^{\pi} f_{2}(t)\left(x^{\prime}(t)\right)^{2} d t, \quad(D g)(t)=g^{\prime}(t) .
\end{gathered}
$$

Then, for any $x \in E$,

$$
\Delta^{L} F_{1}(x)=2 \int_{0}^{\pi} f_{1}(t) d t
$$

and, therefore, by the preceding proposition,

$$
\Delta_{D^{-1}}^{L} F_{2}(x)=2 \int_{0}^{\pi} f_{2}(t) d t
$$

(at the same time, $\Delta_{D^{-1}}^{L} F_{1}(x)=0$ for all $x$ and $F_{2}$ does not belong to the domain of the operator $\Delta^{L}$ ).

\section{LAPLACIANS AND QUANTUM RANDOM PROCESSES}

By definition, a quantum random process is a function defined on a part of the real line and taking values in some space of operators. ${ }^{2}$ In what follows, it is not necessary to choose a particular space of this kind.

\footnotetext{
${ }^{2}$ For example, in Hida calculus (which is also known as white noise analysis), these operators act from a space of test functions defined on a suitable space of generalized functions endowed with the Gaussian measure to a space of distributions (on the space of generalized functions). The relationship between the classical (Kolmogorov) theory of random functions, the GelfandIto theory of generalized random functions, and Hida calculus can be described as follows. To pass from a classical random function, that is, a function $f$ of two arguments (time and an element of the probability space) to a generalized random function, we must allow $f$ to be a generalized function of the first argument, and to pass to a random function in the sense of Hida calculus, we must allow $f$ to be generalized in the second argument (and, in addition, assume the probability space to be chosen in a special way). Any ordinary random function locally integrable with probability 1 can be identified with a generalized random function (over the space D), but not any generalized random function can be assigned a value at every moment of time (an example is the derivative of a Wiener process). In Hida calculus, any random process (including all derivatives of a Wiener process) has values at every moment of time, but these values are distributions on an infinite-dimensional vector space (with Gaussian measure) rather than random variables, and not every ordinary random function can be assigned to a random process in the sense of Hida.
} 
For $h \in E^{*}$, by $b(h)$ we denote the operator of differentiation in the direction $h$, which acts on a function space on $E$. Thus, if $z \in C_{2}(E)$ and $x \in E^{*}$, then $b(h)(z)(x)=z^{\prime}(x) h$.

In what follows, we assume that the assumptions of Example 2 hold. If $h=\delta_{t}$, then we write $b(t)$ instead of $b(h)$; if $h$ is the shift $\theta_{t}$ by $t$ of the Heaviside function $\theta$, then we write $b_{\theta}(t)$ instead of $b(h)$.

The symbols

$$
\begin{gathered}
\int_{0}^{\pi} \delta_{t} \otimes \delta_{t} d t^{2} \equiv \int_{0}^{\pi} \otimes \delta_{t}^{2} d t^{2}, \\
\int_{0}^{\pi} \otimes \theta_{t}^{2} d t^{2}, \quad \int_{0}^{\pi} b(t)^{2} d t^{2} \equiv \int_{0}^{\pi} b(t) b(t) d t^{2}, \quad \int_{0}^{\pi} b_{\theta}(t)^{2} d t^{2}
\end{gathered}
$$

are defined by the equalities (here and in what follows, we consider integrals of functions taking values in spaces of generalized functions or of operator-valued functions on $[0, \pi]$ )

$$
\begin{aligned}
& \int_{0}^{\pi} \otimes \delta_{t}^{2} d t^{2}=\lim _{\epsilon \rightarrow 0} \int_{\{|t-s|<\epsilon ; t, s \in[0, \pi]\}} \delta_{t} \otimes \delta_{s} d t d s, \\
& \int_{0}^{\pi} \otimes \theta_{t}^{2} d t^{2}=\lim _{\epsilon \rightarrow 0} \int_{\{|t-s|<\epsilon ; t, s \in[0, \pi]\}} \theta_{t} \otimes \theta_{s} d t d s, \\
& \int_{0}^{\pi} b(t)^{2} d t^{2}=\lim _{\epsilon \rightarrow 0} \int_{\{|t-s|<\epsilon ; t, s \in[0, \pi]\}} b(t) b(s) d t d s,
\end{aligned}
$$

and

$$
\int_{0}^{\pi} b_{\theta}(t)^{2} d t^{2}=\lim _{\epsilon \rightarrow 0} \int_{\{|t-s|<\epsilon ; t, s \in[0, \pi]\}} b_{\theta}(t) b_{\theta}(s) d t d s .
$$

We also use other similar notations.

For each positive integer $n$, the symbols $b^{(n)}(t)$ and $\delta_{t}^{(n)}$ denote the derivatives of the corresponding orders of the functions $b(\cdot)$ and $\delta_{(\cdot)}$; we set $b^{0}(t)=b(t)$ and $\delta_{t}^{(0)}=\delta_{t}$; for each negative integer $n$, we define $b^{(n)}(t)=$ $\int_{0}^{t} b^{(n+1)}(\tau) d \tau$ and $\delta_{t}^{(n)}=\int_{0}^{t} \delta_{\tau}^{(n+1)} d \tau$ (in particular, $\left.b^{(-1)}(t)=b_{\theta}(t)\right)$.
Proposition 4. For any integer n,

$$
\int_{0}^{\pi} b^{(n)}(t)^{2} d t^{2}=\int_{0}^{\pi} \delta_{t}^{(n)} \otimes \delta_{t}^{(n)} d t^{2} \circ d^{2}
$$

in particular,

$$
\int_{0}^{\pi} b_{\theta}(t)^{2} d t^{2}=\int_{0}^{\pi} \theta_{t} \otimes \theta_{t} d t^{2} \circ d^{2},
$$

where dis the Gáteaux differentiation operator.

Theorem 1. For any integer $n$,

$$
\Delta_{D^{n}}=\int_{0}^{\pi} b^{(n)}(t) b^{(n)}(t) d t
$$

in particular,

$$
\Delta_{D^{-1}}=\int_{0}^{\pi} b_{\theta}(t)^{2} d t
$$

Theorem 2. For any integer n,

$$
\Delta_{D^{n}}^{L}=\int_{0}^{\pi} b^{(n)}(t) b^{(n)}(t) d t^{2}
$$

in particular,

$$
\Delta_{D^{-1}}^{L}=\int_{0}^{\pi} b_{\theta}(t)^{2} d t^{2}
$$

Remark 6. If the space $H$ is endowed with the canonical Gaussian measure, then the functions $t \mapsto \theta_{t}$ and $t \mapsto \delta_{t}$ describe a Wiener process and its derivative, i.e., the so-called white noise. If the spaces on which the operators $b_{\theta}(t)$ and $b(t)$ act and the adjoint operators $\left(b_{\theta}(t)\right)^{*}$ and $(b(t))^{*}$ are defined in an appropriate way, then the functions $t \mapsto\left(b_{\theta}(t),\left(b_{\theta}(t)\right)^{*}\right.$ and $t \mapsto(b(t)$, $(b(t))^{*}$ are a quantum Wiener process and a quantum white noise, respectively (cf. [10]).

Thus, in the situation under consideration, the representations of classical Laplacians use quantum white noise, while those of nonclassical Laplacians use its derivatives or integrals, in particular, the Wiener process.

Theorems 1 and 2 contain results obtained of [4] on representations of classical Laplacians (they correspond to $n=0$ ).

DOKLADY MATHEMATICS Vol. $76 \quad$ No. 32007 


\section{ACKNOWLEDGMENTS}

O.G. Smolyanov acknowledges the support of the Russian Foundation for Basic Research (project no. 0601-00761a) and the Ministry of Education and Science of Spain (grant no. SAB2005-0200).

\section{REFERENCES}

1. I. Ya. Aref'eva and I. V. Volovich, in Proceedings of the International Conference "Generalized Functions and Their Applications in Mathematical Physics," Moscow, Russia, 1981 (Vychisl. Tsentr Akad. Nauk SSSR, Moscow, 1981).

2. L. Accardi, P. Gibilisco, and I. V. Volovich, Russ. J. Math. Phys. 2, 235-250 (1994).
3. L. Leandre and I. V. Volovich, Infinite Dimens. Analysis, Quantum Probab. Relat. Top. 4 (2), 151-172 (2002).

4. L. Accardi and O. G. Smolyanov, Dokl. Math. 65, 356362 (2002) [Dokl. Akad. Nauk 384, 295-301 (2002)].

5. L. Accardi and O. G. Smolyanov, Dokl. Akad. Nauk 342, 442-446 (1995).

6. L. Accardi and O. G. Smolyanov, Conf. Sem. Univ. Bari 250, 1-25 (1993).

7. L. Accardi, O. G. Smolyanov, and M. O. Smolyanova, Math. Notes 60 (1-2), 212-215 (1997) [Mat. Zametki 60 (2), 288-292 (1996)].

8. H.-H. Kuo, N. Obata, and K. Saito, J. Funct. Anal. 94, 74-92 (1990).

9. L. Accardi and N. Obata, in White Noise Analysis and Quantum Probability (RIMS Kokyoroki, Kyoto, 1994), pp. 8-19.

10. N. Obata, Nonlinear Anal. 47, 2437-2448 (2001). 